\title{
THE BOUNDARY PROBLEM ASSOCIATED WITH A DIFFERENTIAL SYSTEM RATIONAL IN THE PARAMETER*
}

\author{
BY \\ RUDOLPH E. LANGER
}

1. Introduction. It is the purpose of this paper to consider the boundary problem associated either with the ordinary differential equation

$$
u^{\prime \prime}(x)+P_{1}(x, \lambda) u^{\prime}(x)+P_{2}(x, \lambda) u(x)=0,
$$

or with the vector differential equation of the second order

$$
U^{\prime}(x) \cdot-2(x, \lambda) U(x) \cdot=\mathcal{O},
$$

when the coefficients involved both in the equation and the adjoined boundary conditions are suitably restricted rational functions of the parameter. For the more familiar case in which the parameter enters the system in polynomial form an extensive theory is at hand, and this theory, together with the available asymptotic forms of solutions of the equation, is of direct application in the present discussion.

The extension to the case of coefficients rational in $\lambda$ for the case of an equation of the first order has previously been made $\dagger$, and that simpler discussion is typical of the present one in that the differential system is found, very naturally, to exhibit in the neighborhood of a pole of the coeffcients the properties which are peculiar to the vicinity of $\lambda=\infty$ when the parameter occurs in the form of polynomials. Thus, for instance, any neighborhood of such a pole is found with proper hypotheses to contain infinitely many characteristic values. Roughly speaking, these values are, therefore, more numerous than in the classical case, and accountable to this fact a single determination of coefficients is found to be possible to serve formally for the simultaneous expansion of several "arbitrary" functions in series of solutions of the system.

The present paper is based extensively upon a recent paper by the author $\ddagger$ devoted to the case in which the coefficients are $\lambda$-polynomials. In fact the deductions are as a rule not carried beyond a point at which the facts estab-

* Presented to the Society, September 7, 1928; received by the editors August 2, 1929.

† Langer, R. E., these Transactions, vol. 25 (1923), p. 155.

$\ddagger$ Langer, R. E., these Transactions, vol. 31, pp. 868-906. This paper will be referred to in the text by the designation $\mathrm{L}$. The reader is referred to this paper for references to the literature. 
lished in the paper cited become available. The vector form of the differential system is first shown to be the more general and is then made the primary subject of consideration.

2. The differential equation. The basic hypothesis on the structure of the equation (1) with which we are concerned is that the coefficients $P_{j}(x, \lambda), j=1,2$, be rational functions of $\lambda$ with poles independent of $x$ and of order $j$. If these poles are designated in the complex $\lambda$ plane by $a_{h}, h=1$, $2, \cdots, m,{ }^{*}$ the explicit form of the coefficients is

$$
\begin{aligned}
& P_{1}(x, \lambda)=p_{10}(x)+\sum_{h=1}^{m} \frac{p_{11}^{h}(x)}{\lambda-a_{h}}, \\
& P_{2}(x, \lambda)=p_{20}(x)+\sum_{h=1}^{m}\left\{\frac{p_{22}^{h}(x)}{\left(\lambda-a_{h}\right)^{2}}+\frac{p_{21}^{h}(x)}{\lambda-a_{h}}\right\} .
\end{aligned}
$$

A differential equation of form (1), (3) may always be obtained from the system of two equations of first order

$$
\begin{aligned}
& u_{1}^{\prime}(x)=q_{11}(x, \lambda) u_{1}(x)+u_{2}(x), \\
& u_{2}^{\prime}(x)=q_{21}(x, \lambda) u_{1}(x)+q_{22}(x, \lambda) u_{2}(x),
\end{aligned}
$$

if the coefficients $q_{i j}(x, \lambda)$ are rational in $\lambda$ with poles of the first order at the points $a_{h}$. In fact if we set

$$
q_{i j}(x, \lambda)=q_{i j}^{0}(x)+\sum_{h=1}^{m} \frac{q_{i j}^{h}(x)}{\lambda-a_{h}},
$$

the elimination of $u_{2}(x)$ from the system (4) yields for $u_{1}(x)$ an equation of form (1), (3) with coefficients which may be computed from the formulas

$$
\begin{aligned}
q_{11}^{h}+q_{22}^{h} & =-p_{11}^{h} & & (h=1,2, \cdots, m), \\
q_{11}^{0}+q_{22}^{0} & =-p_{10}, & & \\
q_{11}^{h} q_{22}^{h} & =p_{22}^{h} & & (h=1,2, \cdots, m),
\end{aligned}
$$

(6)

(d) $\begin{array}{r}h q_{22}^{0}+\underset{q_{11}}{h} q_{22}^{0}-q_{21}^{h}+\sum_{l \neq h} \frac{q_{11}^{h} q_{22}^{l}-q_{11}^{l} q_{22}^{h}}{a_{h}-a_{l}}-\left(\begin{array}{r}h \\ q_{11}\end{array}\right)^{\prime}=p_{21}^{h} \\ (h=1,2, \cdots, m) \text {, }\end{array}$

(e) $\quad \stackrel{0}{q_{11} q_{22}^{0}}-\left(\stackrel{0}{q_{11}}\right)^{\prime}-\stackrel{0}{q_{21}}=p_{20}$.

We wish to show conversely that the equation (1), (3) is always expres-

* For the sake of symmetry we assume the poles to be all located in the finite portion of the plane. This involves no loss of generality since it can always be achieved by at most a change of parameter. 
sible in the form of a system (4), (5). This is readily done by establishing as follows the solvability of the system of equations (6) for the quantities $q$ with given values $p$. To begin with the equations (6a) and (6c), determine the quantities $q_{11}^{h}, q_{22}^{h}$ for each fixed value of $h$ as the roots of the equation

$$
\left(q^{h}\right)^{2}+p_{11}^{h}\left(q^{h}\right)+p_{22}^{h}=0 .
$$

In equations $(6 \mathrm{~b})$ and $(6 \mathrm{~d})$ the unknowns are, then, the $(m+2)$ quantities $q_{11}^{0}, q_{22}^{0}$ and $q_{21}^{h}, h=1,2, \cdots, m$, and in these quantities the equations are linear and only $(m+1)$ in number. Lastly the quantity $q_{21}^{0}$ is determined by the equation (6e). It is clear, therefore, since the system (4) is a restricted case of the vector equation, that the theory of the equation (1) is included in that of the equation (2), the elements of the coefficient matrix $2(x, \lambda)$ being given in structure by the formula (5). We shall confine our considerations, therefore, to this latter equation, writing it in the more explicit form

$$
U^{\prime}(x) \cdot-\left\{\sum_{h=1}^{m} \frac{1}{\lambda-a_{h}} 2^{h}(x)+2^{0}(x)\right\} U(x) \cdot=\mathcal{O}
$$

By way of hypotheses the elements of the coefficient matrices in (7) will be assumed to possess such derivatives as the following deductions require, and as an essential feature we shall suppose further that for each value of $h$ the roots $\theta_{1}^{h}(x) \theta_{2}^{h}(x)$ of the equation

$$
\left|\begin{array}{ll}
q_{11}^{h}(x)-\theta^{h} & q_{12}^{h}(x) \\
q_{21}^{h}(x) & q_{22}^{h}(x)-\theta^{h}
\end{array}\right|=0
$$

are distinct, non-vanishing and of constant arguments. If these arguments are suitably determined we have then

$$
\begin{gathered}
\theta_{1}{ }^{h}(x) \neq \theta_{2}{ }^{h}(x), \\
\theta_{j}{ }^{h}(x)=\left|\theta_{j}{ }^{h}(x)\right| e^{i c_{j}} ; j=1,2 ;\left|c_{1}{ }^{h}-c_{2}{ }^{h}\right| \leqq \pi .
\end{gathered}
$$

Without loss of generality we may consider the variable $x$ to range over the interval $(0,1)$.

3. The related polynomial equations. The change of parameter

$$
\rho_{k}=\frac{\beta_{k}}{\lambda-a_{k}}
$$

in which $\beta_{k}$ is any constant, projects the pole $\lambda=a_{k}$ to $\rho_{k}=\infty$ and gives to the equation (7) the form

$$
U^{\prime} \cdot-\left\{\beta_{k}^{-1} \mathscr{2}^{k} \rho_{k}+\left(2^{0}+\sum_{h \neq k} a_{k h}^{-1} \mathscr{Q}^{h}\right)-\sum_{h \neq k} \frac{\beta_{k}}{a_{k h}\left(a_{k h} \rho+\beta_{k}\right)} \mathscr{Q}^{h}\right\} U \cdot=\mathcal{O},
$$


where we have abbreviated by setting

$$
a_{k}-a_{h}=a_{k h} \text {. }
$$

This equation we shall further transform by the change of dependent variable

$$
U(x) \cdot=\Phi_{k}(x) \Upsilon^{k}(x) \cdot
$$

choosing the matrix $\Phi_{k}(x)$, as is always possible, L $\S 3$, so that in the resulting form of the equation, i.e.,

$$
\Upsilon^{\prime k}(x) \cdot-\left\{R^{k}(x) \rho_{k}+\mathbb{B}^{k}(x)+\sum_{h \neq k} \frac{1}{a_{k h} \rho_{k}+\beta_{k}} \mathscr{B}^{k h}(x)\right\} \Upsilon^{k}(x) \cdot=\mathcal{O},
$$

the leading coefficient matrices have the structure

$$
\mathbb{R}^{k}(x)=\left(\begin{array}{cc}
r_{1}^{k}(x) & 0 \\
0 & r_{2}^{k}(x)
\end{array}\right), \quad \mathbb{B}^{k}(x)=\left(\begin{array}{cc}
0 & b_{1}^{k}(x) \\
b_{2}{ }^{k}(x) & 0
\end{array}\right),
$$

with

$$
r_{j}(x)=\theta_{j}(x) / \beta_{k} .
$$

From the hypotheses (8) and relation (12), it follows that the quantities $r_{j}(x)$ are distinct from each other and from zero, and that they are of constant arguments depending upon the value $\beta_{k}$. The particular choice

$$
\beta_{k}=\exp \left(\frac{i}{2}\left(c_{1}{ }^{k}+c_{2}{ }^{k}\right)\right)
$$

which we shall now make is found directly then to lead to the following relations valid for $x$ on the interval $(0,1)$, i.e.,

$$
\begin{aligned}
& r_{1}^{k}(x) \neq r_{2}^{k}(x), \\
& r_{1}^{k}(x)=\left|r_{1}^{k}(x)\right| e^{i \alpha_{k}} \\
& r_{2}^{k}(x)=\left|r_{2}^{k}(x)\right| e^{-i \alpha_{k}}, \quad 0 \leqq \alpha_{k} \leqq \pi / 2 .
\end{aligned}
$$

It will frequently be found convenient to use the given equation in the form (11) as well as in the form (7), and for this reason we note here in particular the relation

$$
R^{k}(x)=\frac{1}{\beta_{k}} \Phi_{k}^{-1}(x) 2^{k}(x) \Phi_{k}(x) .
$$

The polynomial equation

$$
\bar{\Upsilon}^{\prime k}(x) \cdot-\left\{R^{k}(x) \rho_{k}+\mathbb{B}^{k}(x)\right\} \bar{\Upsilon}^{k}(x) \cdot=\mathcal{O}
$$


derived from equation (11) by retaining only the principal part of the coeffcient matrix at $\rho_{k}=\infty$, is in so-called normal form, $L \$ 3$, due to the relations (13). We shall call this equation the $k$ th related polynomial equation of the equation given. It is clear that the procedure outlined above for the pole $a_{k}$ may be made to yield for the given equation a unique related normal polynomial equation associated with each of the poles $a_{h}, h=1,2, \cdots, m$.

The equation adjoint to (7) may be written

$$
\cdot U^{\prime}(x)+\cdot U(x)\left\{\sum_{h=1}^{m} \frac{1}{\lambda-a_{h}} 2^{h}(x)+2^{0}(x)\right\}=\mathcal{O},
$$

and this is reduced by the transformation

$$
U(x)=\cdot Z^{k}(x) \Phi_{k}^{-1}(x),
$$

and the substitution (9) to the alternative form

$$
\cdot \mathcal{Z}^{\prime k}(x)+\cdot Z^{k}(x)\left\{\mathbb{R}^{k}(x) \rho_{k}+\mathbb{B}^{k}(x)+\sum_{h \neq k} \frac{1}{a_{k h \rho_{k}}+\beta_{k}} \mathbb{B}^{k h}(x)\right\}=\mathcal{O}
$$

For this equation the $k$ th related polynomial equation is evidently

$$
\overline{\mathrm{Z}}^{k}(x)+\cdot \overline{\mathrm{Z}}^{k}(x)\left\{R^{k}(x) \rho_{k}+\mathbb{B}^{k}(x)\right\}=\mathcal{O} .
$$

4. The asymptotic solutions. The asymptotic forms of a pair of solutions of the polynomial equation (15), valid when $\rho_{k}$ is confined to any region in which the quantity

$$
\operatorname{Re}\left(\rho_{k}\left\{r_{1}^{k}(x)-r_{2}^{k}(x)\right\}\right) *
$$

remains either greater than or less than any specific constant and $\left|\rho_{k}\right|$ is sufficiently large, are given, $\mathrm{L} \$ 4$, by the columns of a matrix

$$
\bar{\Upsilon}^{k}(x)=\bar{\Phi}^{k}\left(x, \rho_{k}\right) \varepsilon^{k}\left(x, \rho_{k}\right),
$$

with

$$
\begin{aligned}
e_{i j}^{k}(x, \rho) & =\delta_{i j} e^{\rho_{k} R_{j}^{k}(x)}, \\
\bar{p}_{i j}^{k}(x, \rho) & =\rho_{k}^{\delta_{i j}-1}\left[\bar{p}_{i j}^{k}(x)\right], \quad \bar{p}_{i j}^{k}(x) \equiv 1,
\end{aligned}
$$

the notation being that of the paper cited. A review of the reasoning and manipulation by which these forms are deduced $\dagger$ shows directly that the process requires no essential modification if the considerations are transferred

* $\operatorname{Re}(\omega)$ is used to denote "the real part of $\omega . "$

† Birkhoff and Langer, Proceedings of the American Academy of Arts and Sciences, vol. 58 (1923), p. 51, Sections V and VI. 
from the polynomial equation to the equation (11), the terms in $\left(a_{k n} \rho_{k}+\beta_{k}\right)^{-1}$ being thought of as first expanded in powers of $1 / \rho^{*}$ Moreover, the determination of the initial terms of the asymptotic series is found to be independent of the matrices $\mathcal{B}^{k h}(x)$, and in consequence the equation (15) may be shown to admit of a pair of solutions given asymptotically by the columns of a matrix

$$
\Upsilon^{k}(x)=\mathscr{P}^{k}\left(x, \rho_{k}\right) \varepsilon^{k}\left(x, \rho_{k}\right),
$$

in which the formulas (18b) may be used for $\varepsilon^{k}$ and $\Phi^{k}$ respectively. In entirely similar fashion the rows of a matrix

$$
Z^{k}(x)=\left(\varepsilon^{k}\left(x, \rho_{k}\right)\right)^{-1} \mathcal{X}\left(x, \rho_{k}\right),
$$

with

$$
m_{i j}^{k}\left(x, \rho_{k}\right)=\rho_{k}^{k_{i j}-1}\left[m_{i j}^{k}(x)\right], \quad m_{j i}^{k}(x) \equiv 1,
$$

are found to give the asymptotic forms of a pair of solutions of the adjoint equation (17) or the polynomial equation associated with it.

To insure the availability of these forms over the entire remote portion of the $\rho_{k}$ plane we must make the hypothesis that

$$
\arg \left\{r_{1}^{k}(x)-r_{2}^{k}(x)\right\}=A^{k} \quad \text { (a constant), }
$$

for each value of $k$. The asymptotic forms are applicable then for $p_{k}$ restricted to any half-plane bounded by a line parallel to the ray

$$
\arg \rho_{k}=\pi / 2-A^{k} \text {. }
$$

5. The boundary condition and the characteristic values. As the boundary condition to be imposed upon the given equation (7) we prescribe the relation

$$
\mathscr{W}_{0}(\lambda) U(0) \cdot+\mathfrak{W}_{1}(\lambda) U(1) \cdot=\mathcal{O},
$$

the elements of the coefficient matrices $\mathscr{W}_{0}$ and $\mathscr{W}_{1}$ being any rational functions of $\lambda$ with poles only in the points $a_{h}$. If we suppose the elements of these matrices to be reduced to a common denominator, this denominator is of the form

$$
\prod_{h=1}^{m}\left(\lambda-a_{h}\right)^{\iota_{h}},
$$

in which each exponent $l_{h}$ is a positive integer or zero. Since by equation (7)

* If equation (14) is one which is reducible to a single equation of the second order, the coefficients being written as series in powers of $1 / \rho$, the asymptotic forms are given directly by Birkhoff, these Transactions, vol. 9 (1908), and by Tamarkin, Mathematische Zeitschrift, vol. 27 (1927), p. 1 (and Petrograd, 1917). 
$\lambda$ is restricted from assuming the values $a_{h}$ we may, for any value of $k$, $k=1,2, \cdots, m$, multiply the equation (20) by the quantity

$$
\prod_{l \neq k}\left(\frac{\lambda-a_{h}}{\lambda-a_{k}}\right)^{l_{h}}
$$

without thereby altering it in content. The elements of the new coefficient matrices, however, have poles only at the point $a_{k}$, and under the change of parameter (9) and the substitution (10) the condition takes the form

$$
\mathfrak{W}_{12}^{k}\left(\rho_{k}\right) \Upsilon^{k}(0) \cdot+W_{34}^{k}\left(\rho_{k}\right) \Upsilon^{k}(1) \cdot=\mathcal{O} .
$$

In this the elements of the matrices $W_{j l}^{k}\left(\rho_{k}\right)$ are polynomials in $\rho_{k}$. The values of $\lambda$, if any, which satisfy the system (7), (20) clearly correspond under the relation (9) to the values of $\rho_{k}$ which satisfy the system (11), (21) and vice versa.

The equation (11) was found in $\S 4$ to admit of solutions which for $\left|\rho_{k}\right|$ sufficiently large and $\rho_{k}$ confined to a suitable half-plane are represented asymptotically by the columns of the matrix (19). By familiar reasoning then, $L \S 6$, the characteristic values of the given boundary problem are shown to be the roots of the determinant equation

$$
\left|W_{12}^{k}\left(\rho_{k}\right) \Phi^{k}\left(0, \rho_{k}\right)+W_{34}^{k}\left(\rho_{k}\right) P_{\left(1, \rho_{k}\right)}^{k} \varepsilon^{k}\left(1, \rho_{k}\right)\right|=0 .
$$

We prescribe now for the associated polynomial equation (15) the boundary condition

$$
\overline{\mathscr{W}}_{12}^{k}\left(\rho_{k}\right) \bar{\Upsilon}^{k}(0) \cdot+\overline{\mathscr{W}}_{34}^{k}\left(\rho_{k}\right) \bar{\Upsilon}^{k}(1) \cdot=\mathcal{O},
$$

in which the coefficient matrices are to be determined as finite sums of powers of $\rho_{k}$ to satisfy relations of the type

$$
\begin{aligned}
& \bar{W}_{12}^{k}\left(\rho_{k}\right)=W_{12}^{k}\left(\rho_{k}\right) \mathscr{P}^{k}\left(0, \rho_{k}\right)\left(\bar{\Phi}^{k}\left(0, \rho_{k}\right)\right)^{-1}+\frac{1}{\rho^{s}} \Omega_{12}^{k}\left(\rho_{k}\right), \\
& \bar{W}_{34}^{k}\left(\rho_{k}\right)=W_{34}^{k}\left(\rho_{k}\right) \mathscr{P}^{k}\left(1, \rho_{k}\right)\left(\bar{\Phi}^{k}\left(1, \rho_{k}\right)\right)^{-1}+\frac{1}{\rho^{s}} \Omega_{34}^{k}\left(\rho_{k}\right) .
\end{aligned}
$$

In these relations the matrices $\Omega_{j l}$ are to be series of negative powers of $\rho_{k}$ and $s$ is to be an integer which will be specified later. By at most the multiplication of (23) by a power of $\rho_{k}$ the coefficient matrices can be made polynomials. We shall designate the system (15), (23) as the $k$ th associated polynomial system for the given system (7), (20). 
The characteristic values of the boundary problem (15), (23) are the roots of the equation

$$
\left|\overline{\mathscr{W}}_{12}^{k}\left(\rho_{k}\right) \overline{\mathscr{P}}^{k}\left(0, \rho_{k}\right)+\overline{\mathscr{W}}_{34}^{k}\left(\rho_{k}\right) \bar{\Phi}^{k}\left(1, \rho_{k}\right) \mathcal{E}^{k}\left(1, \rho_{k}\right)\right|=0,
$$

and on expansion of the determinant this equation takes the more explicit form, L $\S 6$,

$$
D_{0}^{k}\left(\rho_{k}\right)+D_{1}^{k}\left(\rho_{k}\right) e^{\rho_{k} R_{1}^{k}}+D_{2}^{k}\left(\rho_{k}\right) e^{\rho_{k} R_{2}^{k}}+D_{3}^{k}\left(\rho_{k}\right) e^{\rho_{k}\left\{R_{1}^{k}+R_{2}^{k}\right\}}=0,
$$

the coefficients being of the structure

$$
D_{r}^{k}\left(\rho_{k}\right)=\rho_{k^{k}}^{k_{r}^{k}}\left[\alpha_{r}^{k}\right], \quad \alpha_{r}^{k} \neq 0 .
$$

Now because of (24) the expansion of the equation (22) will be precisely of the form (25), (26) provided the integer $s$ in (24) is chosen sufficiently large. We suppose such a choice of $s$ to have been made. Inasmuch as the characteristic equations of the given and associated systems are then both of the form (25)* it follows that the characteristic values of the given boundary problem which lie in the remote portion of the $\rho_{k}$ plane are represented asymptotically by those of the associated polynomial problem in $\rho_{k}$. From this we conclude in particular, $\mathrm{L} \$ 7$, that if there exist in the remote $\rho_{k}$ plane enumerably many characteristic values, their distribution is such that a sequence of successively larger contours $C_{n}{ }^{k}, n=n_{1}, n_{2}, n_{3}, \cdots$, may be drawn in the $\rho_{k}$ plane to pass between the characteristic values and to have the following properties: (a) on the contours of the set the quotient of the left member of equation (25) by any one of its terms is uniformly bounded from zero; (b) in the annular region bounded by a pair of the contours, $C_{n_{h}}^{h}$ and $C_{n_{l}}^{\mathbf{z}}$ there lie just $\left|n_{h}-n_{l}\right|$ characteristic values.

We shall denote by $\rho_{k s}$ the characteristic values for which $\left|\rho_{k}\right|$ is large, assigning the subscripts so that

$$
\left|\rho_{k s}\right| \leqq\left|\rho_{k, s+1}\right| \text {. }
$$

By means of the substitution (9) the corresponding characteristic values of the system (7), (20) which lie in a neighborhood of $\lambda=a_{k}$ are obtained. These values have the point $a_{k}$ as a limit point. We denote them by $\lambda_{k}$. The substitution (9) similarly transforms the contours $C_{n}^{k}$ into a sequence of decreasing contours $C_{n}^{k}$ surrounding and converging to the point $a_{k}$. We shall find these contours of especial usefulness in the subsequent developments.

If we consider that in the discussion just concluded $k$ may have any of the values $1,2, \cdots, m$, it follows that if each of the associated polynomial

* I.e., they differ at most in the later terms of the quantities $\left[\alpha_{r}{ }^{k}\right]$. 
systems admits of infinitely many characteristic values, then the given boundary problem also admits of infinitely many characteristic values, these values having limit points at and only at the poles of the coefficients. In proceeding we shall suppose that the boundary problem in hand is one in which this situation obtains.

The characteristic solutions of the given equation in the forms (7) and (11) for the value $\lambda=\lambda_{k s}$ we shall denote by $U^{k s}(x) \cdot$ and $\Upsilon^{k s}(x) \cdot$ respectively. In like manner the symbols $\cdot U^{k s}(x)$ and $\cdot Z^{k s}(x)$ shall designate the characteristic solutions of the adjoint equations.

6. The formal expansion of arbitrary vectors. The characteristic values, as shown in the preceding section, have as limit points the $m$ poles of the coefficients. They are, therefore, roughly speaking $m$ times as numerous as in the classical polynomial case, and because of this we shall seek to expand formally not merely a single vector but a set of $m$ vectors arbitrarily given. Thus if the given vectors are denoted by $\mathcal{F}^{h}(x) \cdot h=1,2, \cdots, m$, we shall seek a set of constant coefficients $c_{r}$, to serve simultaneously in the $m$ series developments

$$
\mathcal{F}^{h}(x) \cdot=\sum_{r=1, s=1,2, \cdots, m}^{\infty} \frac{c_{r s}}{\lambda_{r s}-a_{h}} U^{r s}(x) \cdot \quad(h=1,2, \cdots, m) .
$$

The determination of these coefficients requires but little modification of the procedure applied in L \$8. Thus the equations (7) and (16) lead by a familiar procedure to the relation

$$
\begin{aligned}
\int_{0}^{1} U_{p q} \sum_{h=1}^{m} \frac{1}{\left(\lambda_{r s}-a_{h}\right)\left(\lambda_{p q}-a_{h}\right)} 2^{h} U^{r s} \cdot d x \\
\quad+\frac{1}{\lambda_{r q}-\lambda_{p q}}\left\{\left.\cdot U^{p q} U^{r s} \cdot\right|_{0} ^{1}\right\}=\mathcal{O} \text {, for }(p, q) \neq(r, s) .
\end{aligned}
$$

If we suppose then that the $c_{r}$ are in addition to the relations (27) also amenable to a relation of the form

$$
\left.\sum_{r=1, s=1,2, \cdots, m}^{\infty} \frac{c_{r s}}{\lambda_{r s}-\lambda_{p q}} \cdot U^{p q} U^{r s} \cdot\right|_{0} ^{1}=\left\{\cdot U^{p q}(1) \mathcal{C}_{1}+\cdot U^{p q}(0) \mathcal{C}_{0}\right\} K\left(\lambda_{p q}\right) \cdot
$$

in which the $\mathcal{C}_{j}$ are constant matrices and $K\left(\lambda_{p q}\right) \cdot$ is a vector whose specification we defer, we find directly on multiplying the left member of (28) by $c_{r}$ and summing over all values of $r$ and $s$, the equality 


$$
\begin{aligned}
\int_{0}^{1} & U_{p q} \sum_{h=1}^{m} \frac{1}{\lambda_{p q}-a_{h}} 2^{h \mathcal{F}^{h} \cdot d x}+\left\{\cdot U^{p q}(1) \mathcal{C}_{1}+\cdot U^{p q}(0) \mathcal{C}_{0}\right\} K\left(\lambda_{p q}\right) \\
= & c_{p q}\left\{\int_{0}^{1} \mho^{p q} \sum_{h=1}^{m} \frac{1}{\left(\lambda_{p q}-a_{h}\right)^{2}} 2^{h} U^{p q} \cdot d x\right. \\
& \left.\quad+\left[\left.\left(\lambda_{r s}-\lambda_{p q}\right)^{-1} \cdot U_{p q} U_{r s} \cdot\right|_{0} ^{1}\right]_{(r, s)=(p, q)}\right\}
\end{aligned}
$$

Inasmuch as each member of this equation is a matrix in which all elements are the same the relation may be looked upon as formally determining the coefficient $c_{p q}$.

As in $\mathrm{L} \$ 9$ we may utilize the Green's function $G(x, t, \lambda)$ of the given system (7), (20) to derive from the relation (29) a set of contour integrals associated with the formal series (27). Thus we are led by reasoning analogous to that of the paper cited to the relation

$$
\begin{aligned}
\frac{c_{p q}}{\lambda_{p q}-a_{k}} U^{p q}(x)= & \int_{0}^{1} G^{p q}(x, t) \sum_{h=1}^{m} \frac{1}{\left(\lambda_{p q}-a_{h}\right)\left(\lambda_{p q}-a_{k}\right)} \mathscr{2}^{h}(t) \mathcal{F}^{h}(\dot{t}) \cdot d t \\
& +\frac{1}{\lambda_{p q}-a_{k}}\left\{G^{p q}(x, 1) \mathcal{C}_{1}+G^{p q}(x, 0) \mathcal{C}_{0}\right\} K\left(\lambda_{p q}\right) \cdot
\end{aligned}
$$

Further manipulations lead to the association of quantities

$$
\sum \frac{c_{p q}}{\lambda_{p q}-a_{k}} U^{p q}(x) \cdot \sim \mathfrak{J}_{N}\left(x, \cdot \mathcal{F}^{k} \cdot\right)
$$

where

$$
\begin{aligned}
\mathfrak{J}_{N}\left(x, \mathcal{F}^{k} \cdot\right) & =\mathcal{A}^{k}(x) \cdot \\
& +\frac{1}{2 \pi i} \int_{0}^{1} \int_{C_{N}} G(x, t, \lambda) \sum_{h=1}^{m} \frac{1}{\left(\lambda-a_{h}\right)\left(\lambda-a_{k}\right)} 2^{h}(t) \overline{\mathcal{F}}^{h}(t) \cdot d \lambda d t .
\end{aligned}
$$

In these relations the sum in (30) is to extend over those and only those values of $p$ and $q$ for which the point $\lambda_{p q}$ is enclosed by the contour $C_{N}$, and in (31)

$$
\begin{aligned}
& \mathcal{A}^{k}(x) \cdot=x \mathcal{F}^{k}(1) \cdot+(1-x) F^{k}(0) \cdot, \\
& \bar{F}^{k}(x) \cdot=F^{k}(x) \cdot-\mathcal{A}^{k}(x) \cdot .
\end{aligned}
$$

The quantity (31) is the analogue of the quantity $\mathrm{L}(60)$. Its convergence as $C_{N}$ is replaced successively by contours enclosing a greater and greater number of characteristic values corresponds to the convergence of the $k$ th development (27). 
A particular choice of contours $C_{N}$ which is especially useful is that given by the sequence

$$
C_{N}=-c_{n}{ }^{1}-c_{n}{ }^{2}-\cdots-c_{n}{ }^{m},
$$

where the contours $c_{n}^{h}$ on the right are those of the sequences described in $\$ 5 .^{*}$ By this definition $C_{N}$ encloses all those characteristic values which are not enclosed by any of the $c_{n}^{n}$. As $n \rightarrow \infty$ these latter contours converge toward the respective poles $a_{h}$, and the corresponding contours $C_{N}$ accordingly enclose more and more of the characteristic values. We shall suppose in the following discussion that this choice of the contours $C_{N}$ has been made.

A set of quantities $\mathcal{J}_{N}\left(x, F^{k} \cdot\right)$, analogous to those given in $L(65)$, whose convergence corresponds formally to the summability of the developments (27) may also be deduced as in $\mathrm{L} \$ 10$. Thus if we consider the contour $C_{n}^{k}$ of $\$ 5$ to be a circle with radius $\Lambda_{k n}$, we may. define the matrices

$$
S_{k n}(\lambda)=\left(\delta_{i j}\left\{1-\left[\frac{\beta_{k}}{\Lambda_{k n}\left(\lambda-a_{k}\right)}\right]^{\mu_{k}}\right\}^{\sigma_{j k}}\right),
$$

in which the $\sigma_{j k}$ are constants positive or zero and $\mu_{k}$ is determined as in the $k$ th associated polynomial case $\mathrm{L} \$ 13$. The quantities in question may then be written as

$$
\begin{aligned}
\mathscr{J}_{N}\left(x, \mathcal{F}^{k}\right)= & \mathcal{A}^{k}(x) \cdot+\sum_{l=1}^{m} \frac{1}{2 \pi i} \int_{0}^{1} \int_{-C_{n}^{l}} \Phi_{l}(x) S_{l n}(\lambda) \Phi_{l}^{-1}(x) G(x, t, \lambda) \\
& \cdot \sum_{h=1}^{m} \frac{1}{\left(\lambda-a_{h}\right)\left(\lambda-a_{k}\right)} 2^{h}(t) \overline{\mathcal{F}}^{h}(t) \cdot d \lambda d t .
\end{aligned}
$$

It is clear that for $\sigma_{j k}=0, j=1,2 ; k=1,2, \cdots, m$, the matrices (32) reduce to the unit matrix and the quantities (33) reduce to those given by formula (31).

7. The convergence of $\mathcal{J}_{N}\left(x, \mathcal{F}^{k} \cdot\right)$. Let the left members of the equations (7) and (11) be designated respectively by $\mathcal{L}(U \cdot \lambda)$ and $\mathcal{L}_{k}\left(\Upsilon^{k} \cdot \rho_{k}\right)$. Then the equation

$$
\mathcal{L}(U \cdot \lambda)=\Psi(x) \cdot,
$$

with any choice of the vector on the right, is found to be equivalent under the substitutions (9) and (10) to the equation

$$
\mathcal{L}_{k}\left(\Upsilon^{k} \cdot, \rho_{k}\right)=\Phi_{k}{ }^{-1}(x) \Psi(x) \cdot
$$

\footnotetext{
tours $c_{n}{ }^{n}$.

* It is only as a matter of convenience that we write here the same subscript for each of the con-
} 
Now in terms of the Green's function as defined above the solution of equation (34) with condition (20) is given by the formula

$$
U(x) \cdot=\int_{0}^{1} G(x, t, \lambda) \Psi(t) \cdot d t
$$

and if we similarly define $G_{k}(x, t, \lambda)$ as the Green's function of the system (11), (21), the solution of (34a) with the condition (21) is

$$
\Upsilon^{k}(x) \cdot=\int_{0}^{1} G^{k}\left(x, t, \rho_{k}\right) \Phi_{k}^{-1}(t) \Psi(t) \cdot d t
$$

From (35) and (35a) we conclude, therefore, that

$$
G(x, t, \lambda) \equiv \Phi_{k}(x) G_{k}\left(x, t, \rho_{k}\right) \Phi_{k}{ }^{-1}(t) \quad(k=1,2, \cdots, m) .
$$

With this formula we shall transform the quantity (33) by applying on each contour $c_{n}^{l}$ the respective transformation (9). In this manner we obtain in virtue of (14)

$$
\begin{gathered}
\mathcal{J}_{N}\left(x, \mathcal{F}^{k} \cdot\right)=\mathcal{A}^{k}(x) \cdot \\
-\frac{\Phi_{k}(x)}{2 \pi i} \int_{0}^{1} \int_{c_{n}^{k}} S_{k n}\left(a_{k}+\frac{\beta_{k}}{\rho_{k}}\right) G_{k}\left(x, t, \rho_{k}\right) \mathbb{R}^{k}(t) \Phi_{k}^{-1}(t) \overline{\mathscr{F}}^{k}(t) \cdot d \rho_{k} d t \\
-\sum_{(l, h) \neq(k, k)} \frac{\Phi_{l}(x)}{2 \pi i} \int_{0}^{1} \int_{c_{n}^{l}} S_{l n}\left(a_{l}+\frac{\beta_{l}}{\rho_{l}}\right) G_{l}\left(x, t, \rho_{l}\right) \Phi_{l}^{-1}(t) \mathcal{Q}^{h}(t) \overline{\mathcal{F}^{h}}(t) \cdot \\
\cdot \frac{\beta_{l} d \rho_{l}}{\left(a_{l h} \rho_{l}+\beta_{l}\right)\left(a_{l k} \rho_{l}+\beta_{l}\right)} d t .
\end{gathered}
$$

Consider in this formula the Green's functions occurring in the integrands. From the explicit formula, L (73), it is evident that when $\left|\rho_{h}\right|$ is large $G_{h}\left(x, t, \rho_{h}\right)$ is asymptotically given by the Green's function of the $h$ th associated polynomial system. Since the matrix $S_{h n}\left(a_{h}+\beta_{h} / \rho_{h}\right)$ is the same in form as the matrix $\mathrm{L}$ (63), it follows that the first of the integrals in (36) is precisely of the structure of the integral in $L$ (65). The integrand is, therefore, asymptotically the same as $\Phi_{k}(x)$ times the integrand obtained from the expansion of $\Phi_{k}^{-1}(x) F^{k}(x)$. with regard to the $k$ th associated polynomial system, and the convergence of the integral in the latter case implies the convergence of the integral in hand under the same hypotheses.

The integrals occurring under the sign of summation in (36) resemble that just considered with the exception that a factor

$$
\frac{1}{\left(a_{l h} \rho_{l}+\beta_{l}\right)\left(a_{l k} \rho_{l}+\beta_{l}\right)}
$$


occurs in the integrand. Inasmuch as $h, k$, and $l$ are not all equal, this factor is of at least the degree one in $1 / \rho$, and it is clear that the convergence of the integral already considered, for each choice of $k$, to any finite value implies the convergence of the integrals in hand to the value $\mathcal{O}$. We are led, therefore, to state the following

Theorem. The set of expressions $\mathcal{I}_{N}\left(x, \mathcal{F}^{k}\right), k=1,2, \cdots, m$, is equiconvergent with the corresponding set of expressions, $\mathrm{L}$ (65), obtained from the respective expansion of the kth vector of the set $\Im^{h}(x) \cdot, h=1,2, \cdots, m$, with regard to the kth associated polynomial system.

UNIVERSITY OF Wisconsin, Madison, Wis. 